\title{
Die Grammatik fehlt! Fehlt die Grammatik?
}

\author{
Rückwirkungsmechanismen von TestDaF und DSH
}

\section{Christian Krekeler}

Das Angebot an Sprachtests für den $\mathrm{Zu}-$ gang zu einer deutschen Hochschule hat sich erweitert. Neben der Deutschen Sprachprüfung für den Hochschulzugang ausländischer Studienbewerber (DSH) existiert ein zweiter Hochschulzugangstest, der Test Deutsch als Fremdsprache für Studienbewerber (TestDaF). Alternative Prüfungen werden weiterhin als Studienvoraussetzung anerkannt (vgl. Abb. 2 im Anhang): die Zentrale Oberstufenprüfung (ZOP), das Kleine Deutsche Sprachdiplom (KDS), das Große Deutsche Sprachdiplom (GDS) sowie das Deutsche Sprachdiplom der Kultusministerkonferenz, Stufe II (DSD II). Mit den Veränderungen in der Prüfungslandschaft hat sich auch ein neuer Forschungsbedarf ergeben. Ein bislang wenig beachteter Aspekt sind Rückwirkungsmechanismen der unterschiedlichen Sprachtests für den Hochschulzugang. In diesem Beitrag möchte ich die Rolle der Grammatik in der $D S H$ und im TestDaF und spezifische Rückwirkungsmechanismen dieser Tests diskutieren. Die Prüfungen gehen bei der Grammatik unterschiedlich vor: Die DSH (und auch das KDS und die ZOP) enthalten einen expliziten Grammatikteil. Der Prüfungsteil »Verstehen und Bearbeiten wissenschaftssprachlicher Strukturen « der $D S H$ ist verbindlich vorgeschrieben:

»Der Kandidat soll zeigen, daß er wissenschaftssprachlich relevante Strukturen in ei- nem vorgegebenen Text erkennen, verstehen und sie anwenden kann. Die Aufgabenstellung $[\ldots]$ kann u. a. Ergänzungen, Fragen zum Verstehen komplexer Strukturen sowie verschiedene Arten von Umformungen (Paraphrasierung, Transformation) beinhalten.« (Rahmenordnung für die Deutsche Sprachprüfung für den Hochschulzugang ausländischer Studienbewerber, § 9 Abs. 5.4. Zitiert nach: Redaktionsgruppe im Auftrag des FaDaF 2001)

In der »Rahmenordnung für die $D S H «$ wird auch die Anerkennung von TestDaF geregelt. Ein Testteil Grammatik ist nicht vorgesehen:

»Der Test [TestDaF] orientiert sich in seinen Anforderungen an den vorstehenden Regelungen für die $D S H$. Er umfaßt die Teilprüfungen >Leseverstehen<, >Hörverstehen<, sschriftlicher Ausdruck' und >mündlicher Ausdruck «." (Rahmenordnung für die Deutsche Sprachprüfung für den Hochschulzugang ausländischer Studienbewerber, § 11. Zitiert nach: Redaktionsgruppe im Auftrag des FaDaF 2001)

Durch den Verzicht auf den expliziten Prüfungsteil Grammatik kommt TestDaF mit insgesamt vier Prüfungsteilen aus. Der TestDaF gleicht in dieser Hinsicht dem Sprachtest International English Language Testing System (IELTS), der zum Nachweis studienrelevanter Sprachkenntnisse in einigen englischsprachigen Ländern verwendet wird. Andere englische Sprachtests für den Hochschulzu- 
gang, wie der Test Certificate of Proficiency in English (CPE) oder der Test of English as a Foreign Language (TOEFL) enthalten demgegenüber fünf Prüfungsteile, darunter einen Grammatikteil. Ich erwähne die englischen Sprachtests, weil Tests aus anderen Ländern bei der Entwicklung von TestDaF berücksichtigt wurden und weil im Fall von IELTS ebenfalls eine Diskussion um die Existenz eines Grammatikteils geführt wurde (vgl. PerlmannBalme 1997).

Die Rolle eines Grammatikteils werde ich unter zwei Fragestellungen erörtern. Zunächst geht es um die Testkonstruktion: Wozu dient ein expliziter Grammatikteil in einem Sprachtest für den Hochschulzugang? Unter welchen Umständen ist er überflüssig? In einem zweiten Schritt stelle ich die Frage nach der Wirkung: Welche Auswirkungen hat ein expliziter Grammatikteil auf die Lehr-Lernprozesse? Welche Auswirkungen ergeben sich aus einem Verzicht auf die explizite Thematisierung von Grammatik?

\section{Testkonstruktionen mit und ohne Grammatik}

Das Testkriterium von Sprachtests für den Hochschulzugang lautet »ausreichende Sprachkompetenz für die Aufnahme eines Studiums«. Benötigt man einen Grammatikteil, um Aussagen zum Testkriterium zu gewinnen? Wenn ein Sprachtest für den Hochschulzugang ohne einen expliziten Grammatikteil an Qualität verliert, ist er beizubehalten. Wenn der Test ohne einen expliziten Grammatikteil ausreichend reliabel und valide ist, kann man vom Standpunkt der Testkonstruktion darauf verzichten, denn der Test prüft ohnehin das, was geprüft werden soll. Allerdings ist die Konstruktvalidität von Grammatikteilen ein Problembereich, auf den ich näher eingehen möchte.

Sprachtests können als Performanztest oder als Kompetenztest angelegt sein, wobei sich direkte und indirekte Verfahren unterscheiden lassen (vgl. McNamara 1997).

\section{Abbildung 1: Performanztest und Kompetenztest}

\begin{tabular}{|l|l|l|}
\hline \multicolumn{1}{|c|}{ Performanztest } & \multicolumn{1}{|c|}{ Kompetenztest } \\
\cline { 2 - 3 } direkt & $\begin{array}{l}\text { Beispiel: Test der Fähigkeit, ein Bewer- } \\
\text { bungsgespräch in der Fremdsprache zu } \\
\text { führen, in einem (simulierten) Bewer- } \\
\text { bungsgespräch }\end{array}$ & $\begin{array}{l}\text { Beispiel: Test der Grammatikkennt- } \\
\text { nisse in einem Prüfungsgespräch }\end{array}$ \\
\cline { 2 - 3 } indirekt & $\begin{array}{l}\text { Beispiel: Test der Fähigkeit, ein Bewer- } \\
\text { bungsgespräch in der Fremdsprache zu } \\
\text { führen, mit einer schriftlichen Aufgabe }\end{array}$ & $\begin{array}{l}\text { Beispiel: Test der Grammatikkennt- } \\
\text { nisse mit einem Multiple-Choice-Ver- } \\
\text { fahren }\end{array}$ \\
\cline { 2 - 2 }
\end{tabular}

Sprachliche Performanztests wollen in erster Linie eine Antwort auf die Frage bereitstellen, ob die Kandidaten ihre Sprachfähigkeit auch in einer spezifischen Situation anwenden können. Sprachliche Kompetenztests haben die allgemeine Beherrschung von Sprache unabhängig von einer konkreten Anwendungssituation zum Gegenstand. Der
Grammatiktest der DSH prüft den rezeptiven und produktiven Umgang mit wissenschaftssprachlich relevanten Strukturen in einem vorgegebenen Text. Er gehört $\mathrm{zu}$ den sprachlichen Kompetenztests, da zugrundeliegende Fähigkeiten der Sprachbeherrschung unabhängig von einer zukünftigen Sprachverwendungssituation getestet werden. 
Bei direkten Tests kann von der gezeigten Leistung auf die Leistung außerhalb der Testsituation geschlossen werden. Sprachtests für den Hochschulzugang sind eher indirekt oder »semi-direkt«. Der DSH-Grammatiktest geht indirekt vor. Er soll Informationen zum Testkriterium »Sprachkompetenz für die Aufnahme eines Studiums « bereitstellen und nicht zur Fähigkeit, Transformationsaufgaben durchzuführen.

Der DSH-Grammatiktest gleicht als indirekter Kompetenztest den Prüfungsteilen "Structure" (TOEFL) und "Use of English " (Certificate of Proficiency in English). Indirekte Kompetenztests bieten Vorteile im Bereich der Reliabilität und der Objektivität. Ihre Ergebnisse sind in der Regel zuverlässig und Meßfehler können leicht vermieden werden. Die Prüfungsergebnisse sind relativ unabhängig von der Durchführung und den Prüfern. Weitere Vorteile indirekter Kompetenztests: Sie sind ökonomisch, weil sie leicht auszuwerten sind. Ihr Einsatzbereich ist breit, die Interpretation der Ergebnisse ist oft aber unklar, denn Aussagen zur Validität indirekter Kompetenztests sind schwierig. Vor allem die Bestimmung der Konstruktvalidität ist ein Grundproblem indirekter Sprachtests. Die Konstruktvalidität bezieht sich darauf, wie gültig die Interpretation der Testergebnisse in Bezug auf das Kriterium des Tests ist. Wenn sich die Ergebnisse des Prüfungsteils auf das Testkriterium verallgemeinern lassen, ist die Konstruktvalidität des Tests hoch. Je indirekter aber ein Test ist, desto größer ist der Interpretationsbedarf und desto schwieriger ist die Bestimmung der Konstruktvalidität.

Dennoch wird bei Grammatiktests häufig auf eine hohe Validität in Bezug auf Konstrukte wie »allgemeine Sprachfähigkeit« oder »kommunikative Kompetenz « hingewiesen.
»Grammar is far more powerful in terms of generalisability than any other language feature. Therefore grammar may still be the most salient feature to test.« (Davies 1982: 151)

Vor allem wenn es nicht allein um Sprachwissenstests (»Wie bildet man das Perfekt?«), sondern um die Anwendung strukturellen Wissens geht (»Verändern Sie die Sätze nach folgendem Muster«), werden Ergebnisse von Grammatiktests als gültiger Indikator für die Beherrschung von anderen Sprachfähigkeiten interpretiert, wobei die Validität in Abhängigkeit von den Kriterien jeweils neu bewertet werden muß.

Zur empirischen Erhebung der Validität bedient man sich bei indirekten Tests eines Hilfsmittels: In der Erprobungsphase wird ein paralleler Test mit einer nachgewiesen hohen Konstruktvalidität eingesetzt. Sind die Ergebnisse beider Tests statistisch vergleichbar, geht man auch bei dem neuen Test von einer hohen Validität aus. Ohne solche Hilfskonstruktionen sind Aussagen zur Konstruktvalidität kaum möglich. Daher lassen sich aus indirekten Kompetenztests kaum Aussagen zu einer zukünftigen Sprachverwendung ableiten. Wann gilt die Prüfung als bestanden, wann ist die erforderliche Sprachkompetenz für die Aufnahme eines Studiums erreicht? Ab 70\% richtiger Antworten? Oder schon ab 25\%? Man ist auf Vergleiche oder Erfahrungswerte angewiesen (»der Kandidat $X$ mit dem Ergebnis $Y$ hat sein Studium mit Erfolg durchlaufen«). Der Testteil selbst gibt wenig Anhaltspunkte für diese Entscheidung.

\subsection{Fehlt die Grammatik im TestDaF?}

Da beim TestDaF von Anfang an auf einen expliziten Grammatikteil verzichtet wurde, gibt es auch keine Untersuchungen, wie der Testteil die Validität bzw. die Reliabilität des Prüfungsergeb- 
nisses beeinflußt. Im Falle eines englischen Sprachtests für den Hochschulzugang, IELTS, ist die Diskussion um den Grammatikteil während der Pilotphase geführt worden. In der aktuellen Version enthält IELTS ebenso wie der TestDaF keinen Grammatikteil, sondern besteht aus insgesamt vier Prüfungsteilen: Hörverstehen, Leseverstehen, schriftlicher Ausdruck und mündliche Prüfung. In einer Pilotversion war ein Grammatikteil enthalten. Der Grammatikteil bestand aus Aufgaben zu grammatischen Strukturen, zur Textkohärenz und zum Wortschatz. Die Kontextualisierung legte dabei die Auswahl der gestesteten Phänomene fest. Es wurde außerdem Wert darauf gelegt, daß sich die Anforderungen der einzelnen Testteile möglichst nicht überschneiden.

Eine Untersuchung der Teilergebnisse aus umfangreichen Probedurchläufen führte zu der Erkenntnis, daß die Korrelation zwischen den Ergebnissen aus dem Grammatikteil und den Testteilen Leseverständnis und Hörverständnis hoch war. $\mathrm{Ob}$ die Korrelation auch bei weniger kontextualisierten Aufgabentypen in gleichem Maße bestanden hätte und ob ein anders gearteter Grammatiktest zusätzliche Informationen geboten hätte, wurde nicht überprüft. Allerdings hat man die Korrelation mit verschiedenen Leseverständnistests feststellen können. In Bezug auf die Reliabilität stellte man fest, daß ein Verzicht auf den Grammatikteil nicht zu qualitativen Einbußen führte. Dem Grammatikteil wurde zwar eine hohe Reliabilität und auch eine hohe Validität in Bezug auf das Testziel »Sprachkompetenz für die Aufnahme eines Studiums « bescheinigt, doch da er die Ergebnisse anderer Teiltests nur bestätigte, wurde er gestrichen. Es handelte sich um eine Maßnahme zur Verbesserung der Testökonomie, die bis heute
Bestand hat. Man hätte übrigens mit dieser Argumentation auch den Leseverständnistest streichen können! Man entschied sich für dessen Beibehaltung, weil man in einem Sprachtest für den Hochschulzugang einen Verlust an Augenscheinvalidität erwartete (vgl. Alderson 1993; Clapham 2000).

Diese Erfahrungen unterstützen meiner Ansicht nach eine vergleichbare Vorgehensweise für den TestDaF. Obwohl der TestDaF zu keiner Zeit einen Grammatikteil enthalten hat, ist es eine legitime Annahme, daß dieser Testteil auch im Fall des TestDaF mit anderen Teiltests korrelieren würde. Wenn man von dieser Annahme ausgeht, ist der Grammatikteil für TestDaF obsolet, obwohl er ein valider Indikator für das Testkriterium sein könnte. Aus Gründen der Testökonomie ist es vertretbar, auf diesen Testteil zu verzichten.

\subsection{Sind die "Wissenschaftssprachli- chen Strukturen« in der DSH überflüs- sig?}

Ich möchte mit einer kurzen Beschreibung des $D S H$-Grammatikteils beginnen, die sich auf die Erläuterungen im $\mathrm{DSH}$ Handbuch stützt. Damit wird allerdings nur ein Ausschnitt der Prüfungswirklichkeit wiedergegeben, denn die Rahmenordnung für die $D S H$ bzw. die Aussagen im $\mathrm{DSH}$-Handbuch werden häufig unterschiedlich ausgelegt oder sogar mißachtet. Betrachtet man DSH-Prüfungen verschiedener Institutionen, trifft man auf eine bunte Prüfungsvielfalt: Inhalte, Prüfungsteile, Aufgabenstellungen und wahrscheinlich auch Anforderungsprofile sind sehr unterschiedlich. Laut $D S H-$ Handbuch ist jedenfalls ein indirekter Prüfungsteil »Wissenschaftssprachliche Strukturen « vorgesehen, der in allen mir bekannten Prüfungen im Prinzip auch enthalten war. 
»Der Prüfungsteil >wissenschaftssprachliche Strukturen < befasst sich sowohl rezeptiv als auch produktiv mit wissenschaftssprachlich relevanten Strukturen in einem vorgegebenen Text. Er will weder die Kenntnis grammatischer Terminologie erfragen, noch theoretisches Wissen der Kandidatinnen und Kandidaten überprüfen, z. B. Kenntnisse über die Bildung des Passivs. Es soll vielmehr geprüft werden, ob die [...] Strukturen kontextgebunden erkannt, verstanden und angewendet werden können [...].« (Redaktionsgruppe im Auftrag des FaDaF 2001: 7/2)

Mit den Beispielen im DSH-Handbuch wird unterstrichen, daß es sich nicht um einen Sprachwissenstest handeln soll. Die Autorinnen und Autoren sehen einen kontextualisierten Sprachverwendungstest vor.

»Die Aufgabenstellung lautet: >Füllen Sie die Lücken aus, ohne den Sinn des Textes zu verändern!«« (Redaktionsgruppe im Auftrag des FaDaF 2001: 7/2)

Hintergrund dieser eindeutigen Aussage dürfte die Beobachtung sein, daß häufig linguistische Fachbegriffe in den Aufgabenstellungen verwendet werden, was der Zielsetzung "grammatische Terminologie wird nicht erfragt « jedoch widersprechen würde. Andererseits stellt diese Empfehlung eine deutliche Einschränkung der »Rahmenordnung für die $D S H$ « dar, welche Aufgabenstellungen in Form von »Ergänzungen, Fragen zum Verstehen komplexer Strukturen sowie verschiedene Arten von Umformungen (Paraphrasierung, Transformation)« vorsieht. Wie dem auch sei: Mit der Aufgabenstellung kann sicherlich eine Reihe von grammatischen Phänomenen erfaßt werden. Sie wird im Handbuch anhand eines Texts zum Thema »Landschaftsschutz« erläutert:

»Füllen Sie die Lücken aus, ohne die Textinformation zu verändern! Die Unterstreichungen sollen Ihnen bei der Lösung helfen:
Fragt man nach den Fragt man, warum in Ursachen der in Deutschland in den Deutschland in den letzten Jahren verletzten Jahren ver- stärkt Uberschwemstärkt auftretenden mungen auftreten, Überschwemmungen,

so findet man einen so findet man einen wesentlichen Faktor wesentlichen Faktor in der Flurbereini- in der

gung, die in den siebziger und achtziger Jahren im Zuge Flurbereinigung. der EG-Agrarpolitik vorgenommen wurde.

Die Flurbereinigung Die Flurbereinigung ist ein Eingriff in die ist ein Eingriff in die Landschaft, der ihre Landschaft, der natürliche Beschaffenheit nachhaltig verändert.

...

(Redaktionsgruppe im Auftrag des FaDaF 2001: 7/4; Lösungen siehe Anhang)

Insgesamt werden elf verschiedene Strukturen genannt, von denen maximal zehn im Prüfungsteil »Wissenschaftssprachliche Strukturen « thematisiert werden. Dazu zählen Relativsätze, Indirekte Rede, Passiv und Nominalisierungen. Jede Struktur soll nicht mehr als zweimal vorkommen.

Ist ein derartiger Grammatikteil in der $D S H$ überflüssig? Die Erfahrungen von IELTS und TestDaF können nicht direkt auf die $D S H$ übertragen werden. Die $D S H$ findet unter anderen Voraussetzungen statt, so daß unterschiedliche Vorgehensweisen legitim oder sogar notwendig sind. Die Annahmen, die zum Verzicht auf den expliziten Grammatikteil geführt haben, und der damit verbundene Nutzen treffen nicht in gleichem Maße auf die DSH zu.

Erstens ist die Übertragung der Untersuchungsergebnisse der IELTS-Studie auf die $D S H$ fragwürdig. Der Grammatikteil der Pilotversion von IELTS gleicht dem 
Testteil »Wissenschaftssprachliche Strukturen « der DSH zwar durch die Vorgabe der Kontextualität. Allerdings liegt der Schwerpunkt bei der DSH auf grammatischen Strukturen, so daß die Untersuchungsergebnisse nicht direkt übertragen werden können. Weitere Einschränkungen zur Übertragbarkeit der IELTS-Studie auf die $D S H$ folgen im Kapitel 2.1.

Zweitens trifft die Interpretation der Ergebnisse im Fall des DSH-Grammatikteils nicht auf die typischen Schwierigkeiten wie bei anderen indirekten Kompetenztests. Die DSH wird dezentral von »engagierten und erfahrenen Sprachlehrerinnen und -lehrern vorgenommen", welche die Studiensituation in den verschiedenen Fachbereichen überblicken und viele Kandidaten aus dem Unterricht kennen (Wintermann 1998: 110). Aufgrund ihrer Erfahrungen sind sie möglicherweise in der Lage, zu interpretieren, mit welchem Ergebnis ein Teiltest als bestanden gilt.

Drittens dürfte das Argument der Testökonomie im Fall der DSH nicht in gleichem Maße zum Tragen kommen. Häufig werden Grammatikteile gerade wegen der ökonomischen Vertretbarkeit in Sprachtests aufgenommen, sie lassen sich z. B. problemlos auswerten. Der TestDaF wird von einem Team zentral gestellt und ausgewertet. In die Erstellung kann mehr Sorgfalt einfließen, als bei dezentralen Prüfungen. Der TestDaF kann beispielsweise mittels aufwendiger Probedurchläufe geeicht werden. Bei der dezentralen DSH können allenfalls kleine Kontrollgruppen gefunden werden, Erprobungen des Tests stoßen auf organisatorische Probleme. Unter diesen Umständen wäre ein Verzicht auf einen Testteil, der einfach zu bewerten ist, mit dem Hinweis auf die Testökonomie fragwürdig.

\subsection{Zwischenergebnis}

Beim TestDaF läßt sich der Verzicht auf einen expliziten Testteil Grammatik vom
Standpunkt der Testkonstruktion rechtfertigen. Bei einem vergleichbaren Sprachtest (IELTS) wurde eine hohe Korrelation zwischen den Ergebnissen aus einem kontextualisierten Grammatikteil mit den Testteilen Hörverstehen und Leseverstehen beobachtet. Durch den Verzicht ergibt sich ein Gewinn an Ökonomie.

Vom Standpunkt der Testkonstruktion besteht kein Anlaß, den Testteil »Wissenschaftssprachliche Strukturen " in der DSH ebenfalls zu streichen, obwohl er möglicherweise überflüssig ist. Im Fall der dezentralen $D S H$ greift der Gewinn an Ökonomie nicht in gleichem Maße. Außerdem kann die eigentlich schwierige Interpretation der Testergebnisse eines indirekten Kompetenztests von erfahrenen Prüferinnen und Prüfern vor Ort möglicherweise besser geleistet werden.

Das Zwischenergebnis läßt einen wichtigen Faktor außer acht: die Rückwirkungsmechanismen von Sprachtests für den Hochschulzugang.

\section{Tests wirken, aber wie?}

Auch die Auswirkungen eines Tests auf die Lehr- und Lernprozesse sind ein Qualitätsmerkmal. In der englischsprachigen Forschung spricht man von »impact« oder präziser von »washback« und »backwash «. »Washback/backwash « werden unterschiedlich abgegrenzt: Einige Autoren beziehen »backwash « auf die allgemeine Pädagogik und »washback « auf die Sprachen, bisweilen wird "washback « weiter auf die britische Angewandte Linguistik eingeschränkt. Manchmal werden beide Begriffe auch synonym verwendet. In der deutschsprachigen Forschung greift man häufig auf die englischen Begriffe zurück oder spricht vom »Backwash-Effekt«. Auch deutsche Termini wie etwa »Rückkopplungsphänomen «, „Rückwirkungsmechanismus « oder »Rückstromeffekt « werden verwendet (vgl. Alderson/Wall 
1993; Bailey 1996; Grotjahn 2000; Schifko 2001; Wall 2000).

Daß Tests Rückwirkungen auf Lehr- und Lernprozesse haben können, ist unbestritten. Häufig wird die Existenz von Rückwirkungsmechanismen als ein Naturgesetz angesehen. Die breite Zustimmung verwundert nicht, denn ein $\mathrm{Zu}$ sammenhang zwischen einem Test und der Vorbereitung darauf erscheint plausibel. Andererseits handelt es sich um einen äußerst vagen Begriff: Welche Auswirkungen sind gemeint? Auswirkungen auf die Lernenden, auf die Lehrenden, auf die Einstellung zum Lernen, auf die Inhalte, auf die Methoden, auf das Curriculum, auf die Gestaltung der Lehrwerke? Sind die Auswirkungen auf alle Lerner, auf alle Lehrer gleich stark?

Im Zusammenhang mit der Diskussion um den Grammatikteil interessieren mich vor allem die Auswirkungen auf die Lernenden: In welchem Ausmaß wird ihre Prüfungsvorbereitung von dem Grammatikteil beeinflußt? Ich werde versuchen, Antworten auf diese Frage aus Prüfungsergebnissen und aus Lehrwerken zur Vorbereitung auf die $D S H$ zu gewinnen.

Zum Nachweis von Rückwirkungsmechanismen und zum besseren Verständnis des Phänomens sind eine Reihe von Studien durchgeführt worden. Man muß jedoch feststellen, daß die empirische Basis zur Beschreibung der konkreten Ausprägung dünn geblieben ist. Das dürfte vor allem an den objektiven Schwierigkeiten liegen, einen empirischen Nachweis zu führen. Und selbst wenn die Untersuchungen zu konkreten Ergebnissen geführt haben, ist die Übertragbarkeit auf andere Lehr-/Lernsituationen fragwürdig: Ist die Studie zu Rückwirkungsmechanismen von japanischen Hochschulzugangsprüfungen, die sich stark von deutschen Prüfungen unterscheiden, für den deutschen Kontext aussagekräftig? (Watanabe 1996) Kann das Verhalten von zwei
Lehrern, die in Hong Kong einen TOEFLVorbereitungskurs erteilt haben - und dabei ganz unterschiedlich vorgegangen sind -, eine Entscheidungshilfe in Bezug auf die DSH bzw. auf TestDaF sein? (Alderson/Hamp-Lyons 1996) Relevant dürften allgemeine Erkenntnisse sein: Diese und andere Studien kommen zu dem Schluß, daß sich zwar Rückwirkungsmechanismen beobachten lassen, sie bei einzelnen Lehrenden und Lernenden aber sehr unterschiedlich ausgeprägt sind. Allein die individuelle Vielfalt derjenigen, die am Lehr-/Lernprozeß beteiligt sind, verbietet demnach vereinfachende Annahmen über Wirkungen und Ursachen. Schließlich stellt der Rückwirkungseffekt nur einen Faktor im komplexen Bedingungsgefüge von Lehr-/Lernprozessen dar. Ob Rückwirkungsmechanismen von deutschen Sprachtests bislang systematisch untersucht worden sind, ist mir nicht bekannt (Forschungsübersichten siehe Alderson/ Wall 1993; Bailey 1996; Wall 2000).

Lassen sich angesichts der Komplexität des Phänomens überhaupt sinnvolle Aussagen zur Rückwirkung von Sprachprüfungen treffen? Zweifel sind angebracht, denn im Fall der Sprachprüfungen für den Hochschulzugang kommt hinzu, daß es sich um eine heterogene Zielgruppe handelt, die uneinheitliche Lernwege zu den Prüfungen beschreitet. Allerdings stellen die Komplexität des Phänomens und Schwierigkeiten beim empirischen Nachweis auch nicht automatisch einen Gegenbeweis dar.

»Diese Prüfung [die DSH] hat großen Einfluß auf den Lehr-Lern-Prozeß in der Prüfungsvorbereitung." (Lee 1998: 4)

Stützen läßt sich die Einschätzung durch die hohe Bedeutung der Sprachtests für den Hochschulzugang.

"If a test is regarded as important, then preparation for it can come to dominate all teaching and learning activities" (Hughes 1989: 1) - »Je höher der Status und je 
gewichtiger die Konsequenzen eines Tests, desto stärker ist sein Backwash-Effekt (statusbezogener Aspekt).« (Schifko 2001: 830).

Sowohl der TestDaF als auch die DSH sind Tests mit gewichtigen Konsequenzen. Die $D S H$ verstärkt ihre Bedeutung über die Besonderheit, daß sie nur einmal wiederholt werden kann. Beim TestDaF tragen nicht zuletzt die Kosten zu einer hohen Augenscheinvalidität bei. Insgesamt scheint jedoch eine große Zurückhaltung bei Aussagen zur Rückwirkung dieser Tests angebracht.

Wenn von Rückwirkungsmechanismen auf den Unterricht die Rede ist, wird damit noch keine Aussage über die Qualität der Rückwirkung getroffen. Der Begriff ist wertfrei. Wann ist eine Rückwirkung als wünschenswert, positiv anzusehen, wann als negativ? Man kann den Standpunkt vertreten, daß Aktivitäten zum Spracherwerb in jedem Fall positiv sind, unabhängig davon, ob sie durch einen bevorstehenden Test ausgelöst wurden oder nicht. Diese Einschätzung muß eingeschränkt werden: Es sollte sich dabei um einen Lerneinsatz handeln, der die Kandidaten auch dem Ziel, der Verbesserung studienbezogener Sprachkenntnisse, näherbringt. Negative Rückwirkungen könnten sich ergeben, wenn Inhalt und Konstruktion des Tests nicht mit den Lehr- und Lernzielen bzw. mit der angestrebten Fertigkeit übereinstimmen. Dann unterscheidet sich die Vorbereitung auf den Test von der Vorbereitung auf die angestrebte Sprachverwendungssituation. Die Lerner eignen sich Fähigkeiten an, mit denen sie zwar den Test, nicht aber in der beabsichtigten Sprachverwendungssituation bestehen können. Wenn die Vorbereitung auf den Test und die Vorbereitung auf die angestrebte Sprachverwendungssituation hingegen identisch sind, kann man von einer positiven Rückwirkung sprechen. Demnach ist bei einem direkten Test eher von einer positiven Rückwirkung auszugehen als bei einem indirekten Test.

$\mathrm{Ob}$ die Rückwirkung eines Grammatiktests als positiv oder negativ angesehen wird, hängt schließlich von der Einschätzung der gewünschten Rolle der Grammatik ab: Ist die Vermittlung von Grammatik ein notwendiger Teil der sprachlichen Studienvorbereitung? Wenn ja, in welcher Form sollte die Vermittlung stattfinden? Ein Blick in Curricula und Lehrund Lernmaterialien macht deutlich, daß sich die Positionen der totalen oder systematischen Immersion nicht durchgesetzt haben, auch wenn man Fremdsprachen ohne eine explizite Grammatikvermittlung lernen kann. Bei der sprachlichen Vorbereitung auf ein Fachstudium gehe ich von dem Konsens aus, daß systematische und kontextualisierte Übungen von Strukturen, die im wissenschaftlichen Sprachgebrauch häufig auftreten, in den studienvorbereitenden Sprachunterricht integriert werden können und sollen. Ein vollständiger Verzicht auf die Vermittlung grammatischer und struktureller Phänomene wäre wenig sinnvoll. Fest steht aber auch, daß die Fähigkeit, Sätze transformieren zu können, nicht das übergeordnete Ziel des studienvorbereitenden Sprachunterrichts ist, sondern allenfalls ein Teilziel, ein Mittel zum Zweck (zur Diskussion um den Grammatikunterricht: Butzkamm 1995; Harden/Marsh 1993; Rall 2001).

Problematisch scheint weniger der Umfang, sondern die Art und Weise der Behandlung von Grammatik zu sein. Wolfgang Butzkamm warnt vor einer einseitigen Konzentration auf eine formorientierte Grammatik im Fremdsprachenunterricht (Butzkamm 1995: 190-193). Seine Einschätzung trifft auch auf den studienvorbereitenden Sprachunterricht zu. Eine Überbetonung der Grammatik legt eine sprachbezogene Kommunikation nahe und kann zu einer Vernachlässigung von 
mitteilungsbezogener Kommunikation im Unterricht führen. Würde man feststellen, daß eine Sprachprüfung für den Hochschulzugang zu einer Fokussierung auf expliziter Grammatikvermittlung bei einer Vernachlässigung kommunikativer Elemente führt, sollte man meiner Ansicht nach von einem negativen Rückwirkungsmechanismus sprechen. Dies wäre ein Argument für eine Änderung der Prüfung.

\subsection{Rückwirkungsmechanismen der $D S H$} Beim DSH-Grammatikteil handelt es sich um einen indirekten Test mit wenig authentischen, aber kontextualisierten Aufgabenstellungen. Die Lösung der Aufgaben wird als Indikator für das Kriterium "Sprachkompetenz für die Aufnahme eines Studiums « interpretiert. Läßt man die
Rückwirkungsmechanismen unberücksichtigt, dürften die Beobachtungen, die im Zusammenhang mit IELTS gemacht wurden, auch auf die DSH zutreffen. Demnach wäre eine hohe Korrelation mit den Prüfungsteilen Textverstehen und Hörverstehen zu erwarten.

Aber unsere Erfahrungen im Studienkolleg der Fachhochschule Konstanz decken sich nicht mit dieser Annahme. Im Gegenteil: Wenn die Ergebnisse einzelner Prüfungsteile stark gestreut sind, ist häufig der Grammatikteil betroffen. Dafür dürften meiner Ansicht nach Rückwirkungsmechanismen verantwortlich sein. Zur Erläuterung möchte ich auf 160 Prüfungsergebnisse von Kandidatinnen und Kandidaten verweisen, die nicht von uns auf die $D S H$ vorbereitet worden waren.

Tabelle 1: Mittelwert und Standardabweichung von DSH-Prüfungsteilen

\begin{tabular}{|lcc|}
\hline & Mittelwert & Standardabweichung \\
\hline Wissenschaftssprachliche Strukturen & 3,094 & 1,3781 \\
Leseverständnis & 3,554 & 1,1312 \\
Textproduktion & 3,242 & 1,2300 \\
Hörverständnis & 3,293 & 1,2081 \\
\hline
\end{tabular}

$\mathrm{n}=160$; Bewertung auf einer Skala von 1,0 bis 5,0; ab 4,1 nicht bestanden

Die Kandidaten haben bei dem Prüfungsteil »Wissenschaftssprachliche Strukturen « besser abgeschnitten als bei den anderen Prüfungsteilen - möglicherweise waren die anderen Prüfungsteile schwieriger. Außerdem fällt auf, daß die Streuung der Ergebnisse etwas größer ist. Der Grammatikteil bringt die unter- schiedlichen Leistungen der Kandidaten also stärker zum Ausdruck als die übrigen Prüfungsteile. Läßt man andere Faktoren außer acht, dann liegt in letztgenanntem Sachverhalt ein Hinweis auf eine hohe Aussagekraft dieses Prüfungsteils - ganz im Gegensatz zur IELTSStudie.

Tabelle 2: Korrelationen (nach Pearson)

\begin{tabular}{||l|c|c|c|c||}
\hline \hline & $\begin{array}{c}\text { Wissenschaftssprachli- } \\
\text { che Strukturen }\end{array}$ & $\begin{array}{c}\text { Lesever- } \\
\text { ständnis }\end{array}$ & $\begin{array}{c}\text { Textpro- } \\
\text { duktion }\end{array}$ & $\begin{array}{c}\text { Hörver- } \\
\text { ständnis }\end{array}$ \\
\hline $\begin{array}{l}\text { Wissenschaftssprachliche } \\
\text { Strukturen }\end{array}$ & $*$ &, 462 &, 486 &, 457 \\
\hline Leseverständnis &, 462 & $*$ &, 626 &, 636 \\
\hline Textproduktion &, 486 &, 626 & ${ }^{*}$ &, 657 \\
\hline Hörverständnis &, 457 &, 636 &, 657 & $*$ \\
\hline
\end{tabular}


Der Korrelationskoeffizient gibt die Beziehung zwischen zwei Ereignissen auf einer Skala von 0 (keine Korrelation) bis 1 bzw. -1 (identische Korrelation) wieder. Die Korrelationen zwischen dem Grammatiktest und den übrigen Testteilen sind deutlich niedriger als die Korrelationen zwischen den übrigen Testteilen unter- einander (vgl. Tab. 2). Während bei IELTS eine hohe Korrelation zwischen Textverständnistests und Grammatiktests festgestellt wurde, kann bei unserer DSH keine Rede davon sein. Um festzustellen, wie es $\mathrm{zu}$ diesem abweichenden Ergebnis kommen konnte, ist ein Blick auf einzelne Prüfungsergebnisse hilfreich.

Tabelle 3: Prüfungsergebnisse mit großer Standardabweichung

\begin{tabular}{|c|c|c|c|c|c|c|c|c|c|c|c|c|c|c|c|}
\hline Kandidat & $A$ & $\bar{B}$ & $\overline{C C}$ & $\overline{D D}$ & $\bar{E}$ & $\overline{F F}$ & $\bar{G}$ & $\mathrm{H}$ & $\overline{I I}$ & $\overline{J J}$ & $\bar{K}$ & $\bar{L}$ & $\bar{M}$ & $\bar{N}$ & $\bar{O}$ \\
\hline Lese & 2 & 1,3 & 0 & 3,2 & 0 & 4,3 & 3,7 & 4,8 & 5,0 & 4,6 & 4,4 & 3,4 & 4,3 & 3,0 & 4,3 \\
\hline Text & 1,2 & 2,2 & 3,7 & 4,8 & 5,0 & 3,6 & 3,5 & 3,5 & 4,2 & 3,3 & 3,0 & 4,2 & 3,7 & 5,0 & 4,4 \\
\hline is: & 2 & 2,3 & 8 & 9 & 4,0 & 5,0 & 2 & 2 & 2 & 0 & 7 & 0 & 4,0 & 4,0 & 3,2 \\
\hline & 5,0 & 4,0 & 2,2 & 1,3 & 1,9 & 1,6 & 1,2 & 1,2 & 1,0 & 1,7 & 1,2 & 1,4 & 1,8 & 1,2 & 1,2 \\
\hline
\end{tabular}

Bewertung auf einer Skala von 1,0 bis 5,0; ab 4,1 nicht bestanden

Für Prüfungsergebnisse mit einer besonders hohen Standardabweichung ist fast ausnahmslos der Grammatikteil verantwortlich. Das Ergebnis der Kandidaten A und B im Prüfungsteil »Wissenschaftssprachliche Strukturen « lag deutlich unter dem Ergebnis der anderen Prüfungsteile. Bei den Kandidaten $\mathrm{C}$ bis $\mathrm{O}$ lag das Ergebnis des Grammatikteils deutlich über demjenigen der übrigen Prüfungsteile. Ich habe einige der Kandidatinnen und Kandidaten in der mündlichen Prüfung kennengelernt. Es stellte sich heraus, daß die Ursache für die Abweichungen zwischen den Ergebnissen der Prüfungsteile in der Vorbereitung lag.

Die Kandidaten A und B hatten sich nicht systematisch auf das Format der DSH vorbereitet. Einer hielt sich seit mehreren Jahren in Deutschland auf, der andere hat seine frühe Kindheit in Deutschland verbracht und ist in der Türkei zur Schule gegangen. Mit den Prüfungsteilen Textverständnis, Hörverständnis, Textproduktion und mit der mündlichen Prüfung hatten diese Kandidaten keine Schwierigkeiten. Doch auf Anforderun- gen des Grammatikteils waren sie nicht vorbereitet.

Die Kandidatinnen und Kandidaten $C$ bis O hatten noch nicht lange Deutsch gelernt. Eine Kandidatin besuchte erst seit vier Monaten einen Sprachkurs. Die Deutschkenntnisse dieser Kandidaten lagen in der Regel unter dem geforderten Oberstufenniveau. Überzeugt haben sie allein bei den »Wissenschaftssprachlichen Strukturen", auf die sie sich gründlich und zielstrebig vorbereitet hatten. Hier liegt eine Ursache für abweichende Prüfungsergebnisse mit IELTS: Im Fall von IELTS handelte es sich um eine Pilotversion des Tests, welcher für die Kandidaten keine Bedeutung hatte. Eine gezielte Vorbereitung auf einzelne Testteile dürfte nicht stattgefunden haben. Mit dem DSH-Grammatikteil sind viele Kandidaten sehr wohl vertraut, denn Format und Inhalt werden in prüfungsvorbereitenden Lehrbüchern detailliert eingeführt.

Diese Beobachtungen sind sowohl mit Blick auf die Konstruktvalidität als auch auf die Rückwirkungsmechanismen bemerkenswert. 
Zur Konstruktvalidität: Die großen Anstrengungen, welche die Kandidaten $C$ bis $\mathrm{O}$ für den Prüfungsteil Grammatik unternommen haben, hatten nur geringe positive Auswirkungen auf die Sprachkompetenz, was an den Ergebnissen der anderen Prüfungsteile deutlich wird. Die Fähigkeiten, die im Prüfungsteil »Wissenschaftssprachliche Strukturen " getestet werden, sind eben nicht direkt auf andere studienrelevante Fertigkeiten übertragbar. Man muß bei einer derart zielgerichteten Vorbereitung davon ausgehen, daß die Konstruktvalidität in Bezug auf das Kriterium »Sprachkompetenz für die Aufnahme eines Studiums « eingeschränkt ist. Die Argumentation unterscheidet sich grundsätzlich von derjenigen bei IELTS: Im Fall von IELTS wurde der Grammatikteil wegen seiner hohen Korrelation mit anderen Prüfungsteilen aus Gründen der Testökonomie gestrichen; bei der DSH ist er fragwürdig, weil die Ergebnisse bei einigen Kandidaten stark abweichen und eine Interpretation in Bezug auf das Testkriterium erschweren. Ob die Ergebnisse des Grammatiktests Interpretationen abseits des Testkriteriums ermöglichen, ist dabei unerheblich, da derartige Überlegungen nicht beabsichtigt sind. (Man könnte beispielsweise auf eine hohe Anstrengungsbereitschaft der Kandidaten schließen, die beim Grammatikteil deutlich bessere Ergebnisse haben.)

Mit Blick auf die Rückwirkungsmechanismen sind die abweichenden Ergebnisse des Grammatikteils ein Hinweis darauf, daß die Grammatik als Selbstzweck ohne Einbettung in kommunikative Zusammenhänge gelernt wurde. Ich halte es für ausgeschlossen, daß eine integrierte Vermittlung von grammatischen Phänomenen zu derartigen Prüfungsergebnissen führen kann. Wenn es in der Prüfungsvorbereitung zu einer einseitigen Konzentration auf die Grammatik kommt, könnte man von einem negativen Rückwirkungsmechanismus sprechen. Dieser ist allerdings nicht allein der Existenz des Grammatikteils zuzuschreiben. Das besondere Problem dieser Kandidatinnen und Kandidaten war die Kluft zwischen dem Anforderungsniveau der Prüfung und ihren sprachlichen Fähigkeiten. In ihrer Not, eine Prüfung bestehen zu müssen, die weit außerhalb ihrer Möglichkeiten lag, haben sie sich auf den Grammatikteil konzentriert. Sie konnten bestimmte Aufgaben isoliert lösen, ein Transfer auf freie Produktionsaufgaben war aber nicht möglich, da ihnen die Voraussetzungen dazu fehlten. Eine einseitige Vorbereitung scheint kein allzu häufiges Phänomen zu sein. Schließlich war nur bei weniger als zehn Prozent der 160 Kandidatinnen und Kandidaten zu beobachten, daß sie sich ohne ausreichenden Zugewinn an kommunikativen Kompetenzen auf den Grammatikteil konzentriert haben. Es ist zwar denkbar, daß sich auch andere Kandidaten einseitig auf den Grammatikteil vorbereitet haben, dann muß es jedoch positive Auswirkungen auf die anderen Prüfungsteile gehabt haben, denn es manifestierte sich nicht in einem auffälligen Prüfungsergebnis. Das Phänomen dürfte nicht zuletzt deshalb so schwach ausgeprägt sein, weil im $D S H$-Handbuch in größter Deutlichkeit eine kontextualisierte Aufgabenstellung gefordert wird. Lehrende und Kandidaten haben kein Interesse daran, die Kontextualisierung aufzuheben. Durch die Verbindung von Textarbeit und Grammatikvermittlung sinkt die Gefahr der Überbetonung der Grammatikarbeit bereits.

Weitere Hinweise auf mögliche Rückwirkungsmechanismen des DSH-Grammatikteils lassen sich aus Lehrmaterialien zur Vorbereitung auf die DSH gewinnen. Die folgenden drei Titel dienen der unmittelbaren Prüfungsvorbereitung. Die 
Kandidaten werden mit dem Format der Prüfung vertraut gemacht und können beispielhaft einige Aufgaben bearbeiten. Die Materialien eignen sich laut Klappentext sowohl für den Selbstlerneinsatz als auch für den Unterrichtseinsatz. Grammatik wird nicht explizit vermittelt, sondern als bekannt vorausgesetzt und lediglich angewendet.

\section{Lodewick, Klaus: DSH \& Studienvorbe-} reitung. Vorbereitung auf ein Studium an einer deutschsprachigen Universität. Göttingen: Fabouda Verlag, 2001.

Die Aufgaben des Übungsbuchs sind jeweils einem $D S H$-Prüfungsteil zugeordnet. Dennoch handelt es sich nicht um eine Sammlung von Musterprüfungen. Es werden Aufgaben und Übungen angeregt, die über die Prüfungsaktivitäten hinausgehen. $\mathrm{Zu}$ den Lesetexten werden beispielsweise Aufgaben zur aktiven Vorentlastung der Texte gestellt. Die prüfungsrelevanten Aufgabenstellungen werden analysiert und schrittweise bearbeitet. So findet auch eine Erweiterung der methodischen Kompetenzen statt. Zum Grammatikteil werden hier auch textuelle Referenzen und Worterklärungen gezählt. Eine dekontextualisierte Konzentration auf Grammatikphänomene findet nicht statt.

Eggers, Dietrich; Müller-Küppers, Evelyn; Wiemer, Claudia; Zöllner, Inge: Prüfungskurs DSH. Vorbereitung auf die Deutsche Sprachprüfung für den Hochschulzugang ausländischer Studienbewerber. Ismaning: Hueber, 1999.

Das Buch hat zwei Hauptteile. Im ersten wird jeder Prüfungsteil anhand eines Beispiels ausführlich erläutert, und es werden Hinweise zur Beantwortung gegeben. Zum Grammatikteil werden mögliche Aufgabentypen ausführlich dargestellt. Im zweiten Hauptteil werden fünf Grammatikteile zur Übung angeboten, die sich durchgängig auf einen Lesetext beziehen. Beispiele für Aufgabenstellungen sind: "Worauf bezieht sich salle (Zeile ...)?« - »Warum wird in Zeile ... der Konjunktiv II benutzt? - »Schreiben Sie die folgenden Sätze um, indem Sie die in Klammern angegebenen Strukturen verwenden « (z. B. Partizipialattribut). Ein inhaltsleeres Abarbeiten der Grammatikaufgaben ist nicht möglich. Das liegt nicht zuletzt daran, daß etwa die Hälfte der Aufgaben eher dem Prüfungsteil Textverstehen zuzurechnen ist.

Jung, Lothar: Deutsche Sprachprïfung für den Hochschulzugang ausländischer Studienbewerber (DSH). Ismaning: Hueber, 1995.

In diesem Titel werden die Prüfungsteile als Gliederungsebenen verwendet. Allerdings finden sich keine Aufgaben zur »Vorgabenorientierten Textproduktion «. Das Kapitel »Wissenschaftssprachliche Strukturen « enthält 15 DSH-typische Grammatiktests, die sich inhaltlich an Lesetexten des folgenden Kapitels (»Bearbeitung von Lesetexten «) orientieren. Die Lerner erhalten einen Schlüssel zu den Übungen. Typische Aufgabenstellungen sind: »Formen Sie die Gliedsätze in Satzglieder um «. - »Ersetzen Sie den jeweils kursiv gedruckten Ausdruck durch ein passendes Modalverb (können, mögen, müssen, wollen) «. - »Formen Sie die Attributsätze (Relativsätze) in Partizipialattribute um«. - »Ergänzen Sie die Präpositionen «. Im Informationsteil wird die Arbeit in Gruppen und eine Bearbeitung mit einem verkürzten Zeitlimit angeregt. Außerdem wird die Arbeit mit einer Grammatik sowie die »Aufarbeitung eines Phänomens" im Unterricht empfohlen (Seite 8).

Allein das letztgenannte Lehrbuch gibt Grund zu der Annahme, daß eine Vorbereitung auf den Grammatikteil der DSH 
bisweilen $\mathrm{zu}$ einer Konzentration auf kontextunabhängiges Sprachwissen verleitet. Diese Annahme wurde durch folgende Erfahrung gestützt: Ich habe einer russischen Studentin ein Exemplar des Buches abgekauft. Alle 15 Grammatiktests waren ausgefüllt, aber nur fünf der 15 Leseverständnistests und nur einige Hörverstehenstests wurden (anhand der Transkriptionen) von ihr bearbeitet. Deutlich wird die Bereitschaft der Studentin, Zeit und Arbeit in die Vorbereitung auf den Grammatikteil zu investieren. Sie hat gewußt, so vermute ich, daß sie sich auf diesen Prüfungsteil am gezieltesten vorbereiten kann. Vielleicht war dieser Prüfungsteil auch besonders angstbesetzt.

Die in der $D S H$ verlangten Transformationen müssen geübt werden. Nicht nur DaF-Lernenden, sondern auch Muttersprachlern dürften die Aufgaben nicht leichtfallen, wenn sie ungeübt sind. Kandidaten, die sich auf die $D S H$ vorbereiten möchten, sind daher gut beraten, sich mit derartigen Aufgabenstellungen $\mathrm{zu}$ beschäftigen. Diese Botschaft nehmen sich einige Kandidatinnen und Kandidaten sehr zu Herzen. Bei den Autorinnen und Autoren von Lehrmaterialien zur Prüfungsvorbereitung scheint die Botschaft der Kontextualisierung der DSH-Grammatikaufgaben insgesamt angekommen zu sein. Sie beziehen den Grammatikteil ein, ohne jedoch die Strukturen dekontextualisiert zu vermitteln.

\subsection{Rückwirkungsmechanismen von TestDaF}

Welche Rückwirkungsmechanismen sind durch den Verzicht auf einen expliziten Grammatikteil beim TestDaF zu erwarten? Nicht zuletzt wegen der vielfachen Bedenken, die das Konzept der Rückwirkungsmechanismen bei näherer Betrachtung ausgelöst hat, sind vorschnelle Schlußfolgerungen zu vermeiden. Der TestDaF setzt mit dem Verzicht auf einen expliziten Grammatikteil ein Signal für einen kommunikativ orientierten Deutschunterricht. $\mathrm{Ob}$ und in welcher Form der Anstoß aufgenommen wird, muß noch untersucht werden. Man muß davon ausgehen, daß Unterrichtende und Lernende ihre Gewohnheiten nicht so schnell ändern. Sie tun gut daran, denn es wäre ungerechtfertigt, aus dem Fehlen eines expliziten Grammatikteils beim TestDaF zu schlußfolgern, sprachliche Strukturen spielten bei dem Test (und im Studium) keine Rolle. Auch wenn ein expliziter Prüfungsteil fehlt, müssen bestimmte Kenntnisse vorhanden sein. Sie werden im TestDaF implizit mitgeprüft und bewertet:

»Der vom Kandidaten realisierte Text wird allerdings anhand von unterschiedlichen Leistungskriterien bewertet wie Gesamtwirkung auf den Rezipienten, Behandlung der Aufgabe (gedankliche Organisation des Textes, Ausführlichkeit, Komplexität, Angemessenheit in Bezug auf Adressat, Situation und Textsorte) und sprachliche Realisierung (z.B. Korrektheit der verwendeten Strukturen, Breite des Wortschatzes, Grad der Textualität, Situationsangemessenheit).« (Grotjahn/Kleppin 2001: 425; Hervorhebung Ch. K.)

Für eine Bewertung der Rückwirkungsmechanismen des TestDaF ist es noch früh. Derzeit ist die Anzahl der Kandidaten, welche den Test absolviert haben, gering. Spezifische Lehrwerke sind noch nicht auf dem Markt und spezielle Sprachkurse zur Vorbereitung auf den TestDaF, die man auswerten könnte, sind noch rar.

\section{Resümee}

Mit den Veränderungen in der Prüfungslandschaft ist auch der Forschungsbedarf gestiegen. Ein wichtiger Aspekt sind Rückwirkungsmechanismen der unterschiedlichen Sprachtests für den Hochschulzugang. In diesem Beitrag habe ich die Rolle des Prüfungsteils Grammatik 
und mögliche Rückwirkungsmechanismen dieses Prüfungsteils diskutiert.

Mir ist nicht bekannt, ob die Entwicklerinnen und Entwickler von TestDaF auch auf andere Testformate wirken wollten. Der TestDaF setzt mit dem Verzicht auf einen expliziten Grammatikteil zweifellos ein Signal für einen kommunikativ orientierten Sprachunterricht. Die Grammatik erhält beim TestDaF den Platz, den sie auch im Fachstudium hat: Sie ist Mittel zum Zweck - und nicht das Ziel. Vielleicht rückt der TestDaF ins Bewußtsein, daß der studienvorbereitende Sprachunterricht nicht der Vorbereitung auf eine Prüfung, sondern der sprachlichen Vorbereitung auf das Studium dient. Mit einer Konzentration auf die studienrelevanten Sprachfertigkeiten ist den Kandidatinnen und Kandidaten meiner Ansicht nach mehr gedient, als mit einer Fixierung auf die Besonderheiten von Prüfungen. Der TestDaF verdeutlicht, daß ein Verzicht auf einen expliziten Grammatikteil möglich ist.

Eine eindeutige Empfehlung läßt sich aus diesen Überlegungen für die $D S H$ nicht ableiten. Bei der DSH gibt es Anzeichen, daß die Existenz des Grammatikteils bei einigen Kandidaten hohe Lernaktivitäten in diesem Bereich auslöst, weil der Prüfungsteil für die Kandidaten leichter auszurechnen ist. In einigen Fällen scheint dies mit einer Vernachlässigung kommunikativer Aktivitäten einherzugehen. Der tatsächliche Nutzen des Grammatikteils mit Blick auf die Konstruktvalidität ist dann eingeschränkt. Grundsätzlich positiv ist die starke Streuung der Ergebnisse des Grammatikteils. Sie kann jedoch wegen einer geringen Konstruktvalidität kaum für eine Differenzierung genutzt werden. Für den Grammatikteil der DSH spricht die Ökonomie der Durchführung, so daß ein Verzicht keine erhebliche Entlastung bringen würde. Schließlich steht die DSH häufig am Ende von studienvor- bereitenden Sprachkursen. Als Abschlußtest ist ein indirekter Kompetenztest wie der Grammatikteil ein vertretbarer Bestandteil eines Sprachtests. Ob die Grammatik unter diesen Umständen weiterhin als ein sinnvoller Prüfungsteil angesehen wird, hängt letztlich auch von der Einstellung zu den notwendigen Grammatikkenntnissen im Studium ab.

\section{Literatur}

Alderson, J. Charles: »The relationship between grammar and reading in an English for Academic Purposes test battery«. In: Douglas, Dan; Chapelle, Carol (Hrsg.): A New Decade of Language Testing Research. Alexandra, VA: TESOL, 1993, 203-219.

Alderson, J. Charles; Hamp-Lyons, Liz: »TOEFL preparation courses: a study of washback", Language Testing 13, 3 (1996), 281-296.

Alderson, J. Charles; Wall, Diane: »Does Washback exist? «, Applied Linguistics 14, 2 (1993), 115-129.

Bailey, Kathleen M.: »Working for washback: a review of the washback concept in language testing «, Language Testing 13, 3 (1996), 257-279.

Butzkamm, Wolfgang: „Unterrichtsmethodische Problembereiche«. In: Bausch, Karl-Richard; Christ, Herbert; Krumm, Hans-Jürgen (Hrsg.): Handbuch Fremdsprachenunterricht. Tübingen; Basel: Francke, 1995, 188-194.

Clapham, Caroline: $\gg$ Assessment for academic purposes: where next? «, System 28 (2000), 511-521.

Davies, Alan: »Language Testing «. In: Kinsella, Valerie (Hrsg.): Surveys 1: Eight State-of-the-art Articles on Key Areas in Language Teaching. Cambridge: Cambridge University Press, 1982, 127-159.

Eggers, Dietrich; Müller-Küppers, Evelyn; Wiemer, Claudia; Zöllner, Inge: Prüfungskurs DSH. Vorbereitung auf die Deutsche Sprachprüfung für den Hochschulzugang ausländischer Studienbewerber. Ismaning: Hueber, 1999.

Grotjahn, Rüdiger: »Testtheorie: Grundzüge und Anwendungen in der Praxis«. In: Wolff, Armin; Tanzer, Harald (Hrsg.): Sprache - Kultur - Politik. Regensburg: 
FaDaF, 2000, 304-341 (Materialien Deutsch als Fremdsprache, 53).

Grotjahn, Rüdiger; Kleppin, Karin: »TestDaF: Stand der Entwicklung und einige Perspektiven für Forschung und Praxis«. In: Aguado, Karin; Riemer, Claudia (Hrsg.): Wege und Ziele: Zur Theorie, Empirie und Praxis des Deutschen als Fremdsprache (und anderer Fremdsprachen). Festschrift für Gert Henrici zum 60. Geburtstag. Baltmannsweiler: Schneider Verlag Hohengehren, 2001, 419-434 (Perspektiven Deutsch als Fremdsprache, 15).

Harden, Theo; Marsh, Clíona: Wieviel Grammatik braucht der Mensch? München: iudicium, 1993.

Hughes, Arthur: Testing for Language Teachers. Cambridge: Cambridge University Press, 1989.

Jung, Lothar: Deutsche Sprachprüfung für den Hochschulzugang ausländischer Studienbewerber (DSH). Wissenschaftssprachliche Strukturen, Bearbeitung von Lese- und Hörtexten sowie Texte und Aufgaben für die mündliche Prüfung. Ismaning: Hueber, 1995.

Lee, Wonkyung: Prüfungen zum Nachweis deutscher Sprachkenntnisse bei ausländischen Studienbewerbern / Studienbewerberinnen (PNdS / DSH): Ihre Praxis und ihr Prüfprofil. Regensburg: Fachverband Deutsch als Fremdsprache (FaDaF), 1998 (Materialien Deutsch als Fremdsprache, 50).

Lodewick, Klaus: DSH \& Studienvorbereitung. Vorbereitung auf ein Studium an einer deutschsprachigen Universität. Göttingen: Fabouda, 2001.

McNamara, Tim F.: »Performance testing «. In: Clapham, Caroline; Corson, David (Hrsg.): Encyclopaedia of language and edu- cation. Vol. 7: Language testing and assessment. Dordrecht: Kluwer, 1997, 131139.

Perlmann-Balme, Michaela: TestDaF. Vorstudie zu dem Projekt »Entwicklung eines Tests Deutsch als Fremdsprache für ausländische Studienbewerber «. München: Goethe-Institut; Bonn: DAAD, 1997.

Rall, Marlene: »Grammatikvermittlung«. In: Helbig, Gerhard; Götze, Lutz; Henrici, Gert; Krumm, Hans-Jürgen (Hrsg.): Deutsch als Fremdsprache. Ein internationales Handbuch zeitgenössischer Forschung. Berlin; New York: de Gruyter, 2000-2001 (Handbücher zur Sprach- und Kommunikationswissenschaft, 19).

Redaktionsgruppe im Auftrag des FaDaF: DSH-Handbuch für Prüferinnen und Prüfer. Münster: FaDaF, 2001.

Schifko, Manfred: »Prüfungen, Zertifikate, Abschlüsse als Planungskategorien für den Unterricht«. In: Helbig, Gerhard u. a. (Hrsg.): Deutsch als Fremdsprache: ein internationales Handbuch. Berlin; New York: de Gruyter, 2001, 827-834 (Handbücher zur Sprach- und Kommunikationswissenschaft, 19).

Wall, Dianne: »The impact of high-stakes testing on teaching and learning: can this be predicted or controlled?«, System 28 (2000), 499-509.

Watanabe, Yoshinori: »Does grammar translation come from the entrance examination? Preliminary findings from classroom-based research «, Language Testing 13, 3 (1996), 319-333.

Wintermann, Bernd: »Zuverlässig - Objektiv - Gültig? DSH und TestDaF - Sprachprüfungen auf dem Prüfstand «, InfoDaF 25, 1 (1998), 104-110. 


\section{Lösungen für die Beispielaufgaben (S. 445):}

...wesentlichen Faktor in der in den siebziger und achtziger Jahren im Zuge der EG-Agrarpolitik vorgenommenen Flurbereinigung. ..., der zu einer nachhaltigen Veränderung / nachhaltigen Veränderungen ihrer natürlichen Beschaffenheit führt.

Abbildung 2: Sprachtests für den Hochschulzugang - Übersicht

\section{Zentrale Oberstufenprüfung (ZOP)}

1) Mündliche Prüfung: Die Einzelprüfung besteht aus zwei Teilen. Teil 1: Vorlesen eines vorbereiteten Textes, Zusammenfassung des Textes und Fragen zum Text, Teil 2: vorbereiteter Kurzvortrag und Gespräch über das Thema. (20 Minuten)

2) Texterklärung eines Lesetextes: ein Zeitungstext oder ein Text aus einem Sachbuch. Aufgaben: Fragen zum Inhalt, zu Bezügen im Text, zum Wortschatz. (90 Minuten)

3) Hörverstehen: Aufgaben zu einem Hörtext, der zweimal gespielt wird (Rundfunksendung, z. B. Bericht oder Feature). (40 Minuten)

4) Aufsatz: eine Aufgabe, Auswahl zwischen Themen, die sich auf eine Lektüre von Büchern beziehen, und freien Themen. (90 Minuten)

5) Ausdrucksfähigkeit: Aufgaben zur Grammatik: Umformungen, Einsetzaufgaben usw., Aufgaben zum Wortschatz. (90 Minuten)

Dauer: 310 Minuten.

Kosten: $€$ 115-150

Ergebnis: Notenstufen (1-3 bestanden)

Träger: Goethe-Institut

Durchführung: Goethe-Institute weltweit und weitere Prüfungszentren.

Ziel: Nachweis allgemeinsprachlicher Kenntnisse.
Kleines Deutsche Sprachdiplom (KDS)

1) Mündliche Prüfung: Die Einzelprüfung besteht aus zwei Teilen. 1. Teil: Vorlesen eines vorbereiteten Texts, 2. Teil: freier Vortrag (Auswahl aus drei Themen) mit anschließendem Gespräch. (Vorbereitungszeit: 40 Minuten, Prüfungsgespräch: 20 Minuten)

2) Erklärung eines Textes nach Inhalt und Wortschatz: Es wird ein literarischer Text vorgelegt. Fragen zum Textinhalt und Aufgaben zum Wortschatz. (90 Minuten)

3) Diktat: Die Kandidaten müssen einen mündlich vorgetragenen Text (20 Zeilen) aufschreiben. (20 Minuten)

4) Aufgaben zur Lektüre: Zwei Aufgaben zu Büchern, die vorbereitet worden sind. 1. Persönliche Stellungnahme zum Inhalt, 2. Erörterung eines Textausschnittes. (120 Minuten)

5) Aufgaben zur Überprüfung der Ausdrucksfähigkeit: Aufgaben zur Grammatik (Umformungen, Einsetzaufgaben usw.), Aufgaben zum Wortschatz. (60 Minuten)

Dauer: 290 Minuten

Kosten: $€ 165-200$

Ergebnis: Notenstufen (1-3 bestanden)

Träger: Goethe-Institut im Auftrag der Ludwig-Maximilians-Universität München.

Durchführung: Goethe-Institute weltweit und weitere Prüfungszentren

Ziel: Nachweis allgemeinsprachlicher Kenntnisse. 
Abbildung 2: Sprachtests für den Hochschulzugang - Übersicht

\section{Deutsche Sprachprüfung für den Hochschul- zugang (DSH)}

1) Mündliche Prüfung: Einzelprüfung $\mathrm{zu}$ allgemein wissenschaftsbezogenen Problemstellungen oder $\mathrm{zu}$ Fragen der gewählten Studienrichtung. (max. 20 Minuten)

2) Leseverstehen: Offene und geschlossene Aufgabenstellungen $\mathrm{zu}$ einem authentischen, studienbezogenen und wissenschaftsorientierten Text. (ca. 45 Minuten)

3) Hörverstehen: Der Hörtext orientiert sich an der Kommunikationssituation Vorlesung/Übung, soll allerdings ohne eine fachspezifische Vorbereitung zu verstehen sein. Er wird zweimal gespielt. Aufgaben zum Inhalt, zur Struktur usw. (ca. 45 Minuten)

4) Vorgabenorientierte Textproduktion: Es wird ein zusammenhängender Text zu einem an Vorgaben gebundenen Thema verlangt. (ca. 45 Minuten)

5) wissenschaftssprachliche Strukturen: Wissenschaftssprachliche Strukturen sollen erkannt, verstanden und angewendet werden. (ca. 45 Minuten)

Dauer: 200 Minuten

Kosten: uneinheitlich, häufig kostenlos

Ergebnis: »bestanden« bzw. »nicht bestanden «

Träger: deutsche Hochschulen

Durchführung: dezentrale Durchführung und Korrektur von Studienkollegs oder Sprachlehrgebieten.

Ziel: Studienzulassung
Test Deutsch als Fremdsprache für Studienbewerber (TestDaF)

1) Mündlicher Ausdruck: Simulated Oral Proficiency Interview - Stimulus wird gehört, gesprochene Antwort wird auf Band aufgenommen. (30 Minuten)

2) Leseverstehen: drei Texte $\mathrm{zu}$ hochschulbezogenen Themen und allgemein-wissenschaftliche Themen. Aufgaben: Zuordnung, dreigliedrige Multiple-Choice-Aufgaben, Auswahlaufgabe (ja/nein/nicht im Text). (60 Minuten)

3) Hörverstehen: drei Hörtexte, die ein- oder zweimal gespielt werden, Aufgabentypen: Stichwörter notieren, richtig-falsch Antworten; gesteuerte Notizen. (30 Minuten)

4) Schriftlicher Ausdruck: zwei Aufgaben: a) Beschreiben von Diagrammen, Tabellen, Abläufen/Vorgängen, b) Argumentation Stellungnahme zu kontroversen Fragen mit Darstellung und Begründung verschiedener Standpunkte. (60 Minuten)

Dauer: 180 Minuten

Kosten: $€$ 90-110

Ergebnis: Qualifiziertes, kriterienorientiertes Ergebnis, das mittels verbaler Deskriptoren ausgewiesen wird. Das Erreichen der TestDaF-Niveaustufe 3, 4 oder 5 wird bestätigt. Stufe 4 entspricht der Zentralen Mittelstufenprüfung, Stufe 5 der Zentralen Oberstufenprüfung bzw. dem Kleinen Deutschen Sprachdiplom.

Träger: Zentrales Testinstitut

Durchführung: Ausrichter im In- und Ausland

Ziel: Studienzulassung 
Abbildung 2: Sprachtests für den Hochschulzugang - Übersicht

\section{International English Language Testing Sys- tem (IELTS)}

1) Mündliche Prüfung: Die Einzelprüfung wird aufgezeichnet. (10-15 Minuten)

2) Leseverstehen: Aufgaben $\mathrm{zu}$ drei Texten (60 Minuten)

3) Hörverstehen: vier kurze Hörtexte mit offenen und geschlossenen Aufgaben (30 Minuten)

4) Schriftlicher Ausdruck: Teil 1: Vorgabenorientierte Textproduktion (Schaubild, Grafik), Teil 2: Argumentation, Stellungnahme usw. (60 Minuten)

Dauer: 125 Minuten

Kosten: $£ 70$

Ergebnis: Qualifizierte Rückmeldung in neun Stufen.

Träger: IELTS

Durchführung: weltweit durch das British Council und andere

Ziel: Zulassungsvoraussetzung an Hochschulen in Australien, Neuseeland, Kanada und Großbritannien. Eine weitere Version richtet sich an Personen, die zur Ausbildung in ein englischsprachiges Land kommen.

Nicht berücksichtigt wurden das Große Deutsche Sprachdiplom (GDS) sowie das Sprachdiplom der Kultusministerkonferenz der Stufe II. Das GDS ähnelt dem KDS, enthält aber Fragen zu einem Fachgebiet (»Deutsche Literatur «, »Naturwissenschaften«
Certificate of Proficiency in English (CPE)

1) Mündliche Prüfung: Einzel- oder Gruppenprüfung, Teil 1: Fotos als Sprechanlaß, Teil 2: Diskussion, Problemlösung usw. (10-15 Minuten)

2) Leseverstehen: Multiple-Choice Aufgaben zum Wortschatz und Inhalt (60 Minuten)

3) Hörverstehen: drei bis vier Sachtexte (30 Minuten)

4) Schriftlicher Ausdruck: zwei Aufsätze (120 Minuten)

5) Strukturen und Wortschatz: Lückentext, Satzvervollständigung, Transformation, Sinnentnahme (120 Minuten)

Dauer: 345 Minuten

Kosten: $£ 40$

Ergebnis: fünf Notenstufen (A-E), bestanden bei A-C.

Träger: University of Cambridge Local Examinations Syndicate (UCLES)

Durchführung: weltweit durch das British Council und andere

Ziel: allgemeine Sprachstandsprüfung, häufig als Zulassungsvoraussetzung anerkannt.

oder »Wirtschaftswissenschaften «) und Fragen zur Landeskunde. Das Sprachdiplom der Kultusministerkonferenz der Stufe II wird an Schulen im Ausland durchgeführt. Zielgruppe sind Schülerinnen und Schüler der Auslandsschulen. 